\title{
The Isotonic Cauchy Transform
}

Ricardo Abreu Blaya, Juan Bory Reyes, Dixan Peña Peña and Frank Sommen

\begin{abstract}
Starting with an integral representation for the class of continuously differentiable solutions $f: \mathbb{R}^{2 n} \rightarrow \mathbb{C}_{0, n}$ of the system

$$
\partial_{\underline{x}_{1}} f+i \tilde{f} \partial_{\underline{x}_{2}}=0 \text {, }
$$

where $\mathbb{C}_{0, n}$ is the complex Clifford algebra constructed over $\mathbb{R}^{n}, \underline{x}_{1}, \underline{x}_{2}$ are some suitable Clifford vectors and $\partial_{\underline{x}_{1}}, \partial_{\underline{x}_{2}}$ their corresponding Dirac operators, we define the isotonic Cauchy transform and establish the SokhotskiPlemelj formulae. Some consequences of this result are also derived.
\end{abstract}

Mathematics Subject Classification (2000). 30G35.

Keywords. Clifford analysis, isotonic functions, Sokhotski-Plemelj formulae.

\author{
Ricardo Abreu Blaya \\ Faculty of Mathematics and Informatics, \\ Universidad de Holguín, \\ Holguín 80100, Cuba. \\ e-mail: rabreu@facinf.uho.edu.cu \\ Juan Bory Reyes \\ Department of Mathematics, \\ Universidad de Oriente, \\ Santiago de Cuba 90500, Cuba \\ e-mail: jbory@rect.uo.edu.cu \\ Dixan Peña Peña \\ Department of Mathematical Analysis, \\ Ghent University, \\ Galglaan 2, B-9000 Gent, Belgium \\ e-mail: dixan@cage.UGent.be
}


Frank Sommen

Department of Mathematical Analysis, Ghent University,

Galglaan 2, B-9000 Gent, Belgium

e-mail: fs@cage.UGent.be

Received: November 11, 2006

Accepted: January 8, 2007 